\title{
A 6I-year-old man presented with myopathy, neuropathy, and inflammatory dermatitis responsive to chronic lymphocytic leukemia treatment
}

\author{
This article was published in the following Dove Press journal: \\ International Journal of General Medicine \\ 30 July 2012 \\ Number of times this article has been viewed
}

\section{Samer Hassan \\ Muhammad Popalzai \\ Edward Yu \\ Monika Wrzolek \\ Marcel Odaimi}

Staten Island University Hospital, Staten Island, New York, NY, USA
Correspondence: Samer Hassan 475 Seaview Avenue, Staten Island University Hospital, Staten Island, New York, NY 10305, USA Email samerhassan83@yahoo.com
Abstract: The prevalence of paraneoplastic neurologic syndrome in cancer is $0.01 \%$. Neurological syndromes can be seen in chronic lymphocytic leukemia (CLL) and mostly present as either leukemic infiltration of the central nervous system (CNS) or progressive multifocal leukoencephalopathy. To our knowledge, this is the first reported case of combined sensorymotor neuropathy, myopathy, and dermatitis in a patient with CLL.

Case presentation: A 61-year-old African American man presented with acute dysphagia, rapidly progressive proximal limb-girdle weakness, and dermatitis. He had a white blood cell (WBC) count of $14,600 / \mathrm{mm}^{3}$, hemoglobin of $11.4 \mathrm{mg} / \mathrm{dL}$, and a platelet count of $165,000 / \mathrm{mm}^{3}$. Lymphocytes comprised $15 \%$ of the total WBC with an absolute lymphocyte count of $2100 / \mathrm{mm}^{3}$. Metabolic profile was unremarkable except for a serum creatine phosphokinase (CPK) level of $1056 \mathrm{mg} / \mathrm{dL}$. Serum protein electrophoresis, serologic studies for autoimmune, genetic diseases, and paraneoplastic syndromes were all negative. Electrodiagnostic studies revealed sensorimotor neuropathy with mixed axonal and demyelinating features. Muscle biopsy revealed discrete areas of interstitial fibrosis juxtaposed to areas of intact muscle without any inflammation. At that point, a bone marrow biopsy was done because of anemia and slightly elevated mean corpuscular volume of 103 . Bone marrow biopsy revealed minimal involvement with CD5/CD19-positive CLL. Flow cytometry demonstrated monoclonal CD5/CD19/CD20/CD23-positive cells, with dim kappa expression, and negative FMC-7 and CD3. This case doesn't meet the criteria for CLL/small lymphocytic lymphoma. However, considering the possibility of paraneoplastic phenomenon for his symptoms, it was decided to start the patient on CLL-directed therapy with Rituximab and Cyclophosphamide. After only two cycles, the patient experienced a dramatic improvement in his muscle strength with disappearance of the rash.

Conclusion: This case highlights a unique clinical picture of inflammatory dermatitis with electromyography and biopsy findings suggestive of myopathy and combined sensorimotor neuropathy with response to CLL-directed therapy. Also the symptoms started before peripheral lymphocytosis which masked the diagnosis for over a year.

Keywords: Chronic Lymphocytic leukemia, paraneoplastic syndrome, sensory-motor neuropathy, myopathy, dermatitis.

\section{Case presentation}

A 61-year-old man African American with a history of diabetes mellitus type 2 and hypertension presented to our clinic with acute dysphagia, proximal limb-girdle weakness, and dermatitis. Dysphagia began two days prior to admission, which was initially described as a "tickling and grabbing" sensation that rapidly progressed to severe dysphagia associated with odynophagia. Symmetric proximal weakness predominantly in 
limb-girdle distribution initially began as weakness with stair climbing and running followed by fasciculation in the thighs and upper arms with fairly rapid progression to inability to stand or squat independently over the course of two months. A rash appeared acutely at the same time as weakness and lethargy, initially affecting the face bifrontally with progression to the entire face, upper torso, and extensor surfaces of the lower extremities eventually becoming confluent.

Physical examination was significant for moderate symmetric proximal weakness of the arms with inability to lift arms above his head. Asymmetric weakness of the legs was present with the right affected more than the left and in both proximal and distal distributions. Fasciculation was apparent in the arms and legs consistent with the distribution of weakness. Asymmetric atrophy was noted predominantly in the legs with midthigh diameters of $39.5 \mathrm{~cm}$ on the right and $44.0 \mathrm{~cm}$ on the left. Deep tendon reflexes were $1+$ in the arms and legs. A macular, nonerythematous skin rash was observed on scalp, face, torso, and extensor surfaces of the limbs.

The patient didn't smoke previously and drinks alcohol socially. He had no history of drug abuse or recent vaccination. His medications included metformin, lansoprazole, Repaglinide, telmisartan, and prednisone, which was started one week prior to the admission for dermatitis.

Complete blood count showed a total white blood cell (WBC) count of $14,600 / \mathrm{mm}^{3}$. Granulocytes comprised $75 \%$ and lymphocytes $15 \%$ of total leukocytes with an absolute lymphocyte count of $2100 / \mathrm{mm}^{3}$. Hemoglobin was $11.4 \mathrm{mg} / \mathrm{dL}$ with a mean corpuscular volume (MCV) of 94 and a platelet count of $165,000 / \mathrm{mm}^{3}$. Metabolic profile was unremarkable except for an albumin of $2.0 \mathrm{mg} / \mathrm{dL}$ and aspartate aminotransferase of $74 \mathrm{mg} / \mathrm{dL}$. Serum creatine phosphokinase level was mildly elevated at $1056 \mathrm{mg} / \mathrm{dL}$.

Initial electrodiagnostic studies revealed nerve conduction consistent with mild length-dependent sensorimotor neuropathy with mixed axonal and demyelinating features. Electromyography revealed denervation potentials and short duration, small amplitude motor units with prominent polyphasia and early recruitment, suggestive of a myopathic process affecting proximal muscles. Subsequent follow-up electrodiagnostic studies over the next month showed progression to widespread denervation with similar myopathic motor unit action potentials with mixed early and reduced recruitment. Serologic studies for autoimmune diseases were negative (eg, rheumatoid factor, RNP antibodies, anti SCL70, anti-Smith, SSA, SSB, anti-ds DNA, smooth muscle antibodies). Serum complement levels were also within normal limits. Genetic testing for survival motor gene (SMN1), androgen receptor CAG repeat expansion, and hexoaminidase deficiency was negative. Serologic testing for GM1 ganglioside autoantibodies was negative. Heavy metal screening for lead, arsenic, and mercury exposure was negative as well. Paraneoplastic antibody screening was negative (eg, anti-Hu, anti-Yo, voltage-gated calcium channel, and anti-Jo antibodies).

A skin biopsy showed superficial perivascular lymphocytic infiltrates with subepidermal neutrophilic infiltrates. Muscle biopsy of the right vastus lateralis revealed two patterns of muscle abnormality. Discrete areas of severe

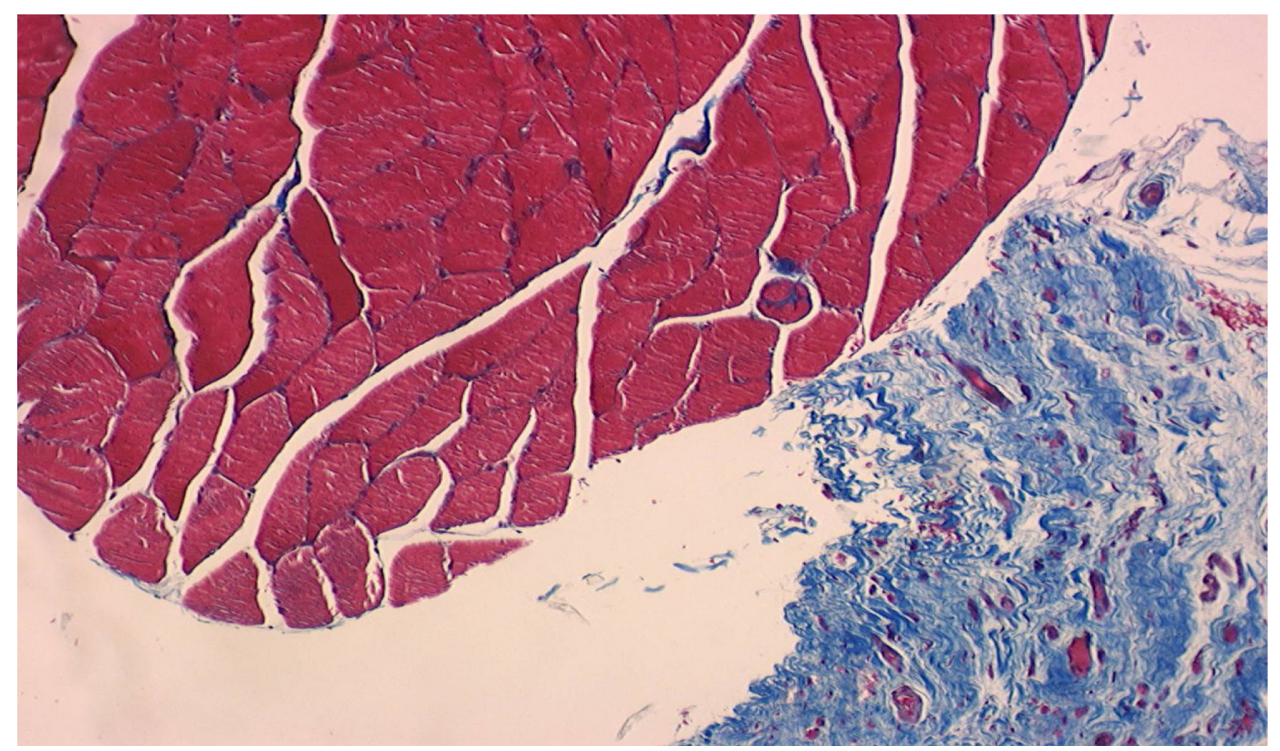

Figure I Trichrome stain showing portion of well-preserved muscle (upper left), and an area of markedly atrophic muscle, with extensive fibrosis (lower right). 
interstitial fibrosis and fatty replacement were juxtaposed to areas of nearly intact muscle (Figure 1; Trichrome stain, low magnification). Adenosine Triphosphatase stain showed prominent fiber-type grouping in the areas with preserved fibers (Figure 2), consistent with a chronic neuropathic pattern of denervation with reinnervation. There was no significant endomysial, perimysial, or perivascular inflammation, and no findings diagnostic of an underlying specific myopathic or dystrophic process.

Magnetic resonance imaging (1.5 Tesla) with intravenous gadolinium diethylenetriaminepentaacetic acid of the neuroaxis revealed chronic mild microvascular ischemic changes in the brain, multilevel degenerative disk disease in the cervical and lumbosacral segments with varying levels of spinal canal and neuroforaminal stenosis. No enhancement of the neuroaxis or nerve roots was seen. Computed tomography with contrast of the chest, abdomen and pelvis revealed few small pulmonary nodules up to $5 \mathrm{~mm}$ in size without any evidence of underlying malignancy in the thorax or intraabdominally. Positron emission tomography (FDGPET w/10.2 mCi 18-fluorine) of the whole body did not reveal any areas of abnormal uptake.

The patient was started on high-dose corticosteroids (prednisone) after initial evaluation for presumed inflammatory myopathy prior to biopsy results with dramatic improvement in the skin lesions and the proximal muscle strength to the point where he was able to stand and walk independently. Despite improvements in limb strength, bulbar symptoms continued to decline to the point where he was intubated for several days due to aspiration. He was subsequently extubated and had percutaneous gastrostomy placed due to severe dysphagia. Macular rash progressed to affect the entire chest, back and flexor surfaces of the limbs both proximal and distal associated with some induration. At that time, patient refused further diagnostic investigation including bone marrow biopsy. After initial response to steroids, limb weakness progressed with subsequent development of atrophy in both proximal and distal muscles. He was eventually started on intravenous immunoglobulin with minimal improvement after 1 month.

At that point it was decided to do a bone marrow biopsy because of anemia and slightly elevated MCV of 103. The examination of the bone marrow biopsy revealed small interstitial lymphoid aggregate (Figure 3 ). The cells within the aggregate were composed of monomorphous small lymphocytes with scant pale cytoplasm and condensed chromatin (Figure 4). Most significantly, the immunophenotype of the lymphoid aggregate expressed CD5 (Figure 5), CD20 (Figure 6), and CD23 (Figure 7), positivity, which was consistent with CLL immunophenotype. Flow cytometry demonstrated monoclonal CD5/CD19-positive cells, CD20 (dim), CD23-positive, kappa (dim), FMC-7-negative, and CD3-negative. It was positive for ZAP 70 and chromosomal analysis showed trisomy 12.

As the absolute lymphocyte count at that time was under $5000 / \mathrm{mm}^{3}$ and there was no lymphadenopathy visible on the imaging, he did not meet the criteria for CLL/small lymphocytic lymphoma. However, considering the possibility of paraneoplastic phenomenon for his symptoms, a diagnosis of CLL in this patient is made with clinical and

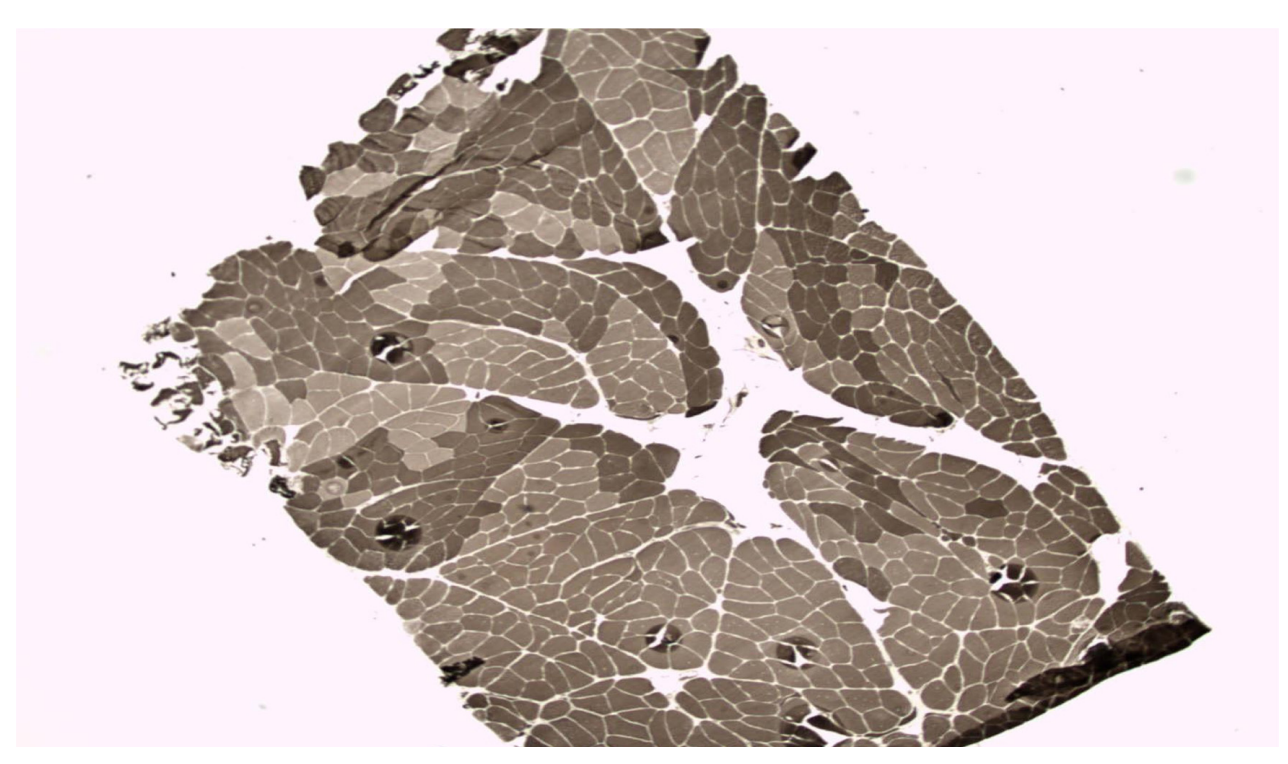

Figure 2 ATP-ase stain showing loss of normal checker-board pattern of muscle fibers, with large groups of type I and type II fibers, diagnostic of denervation/reinnervation. 


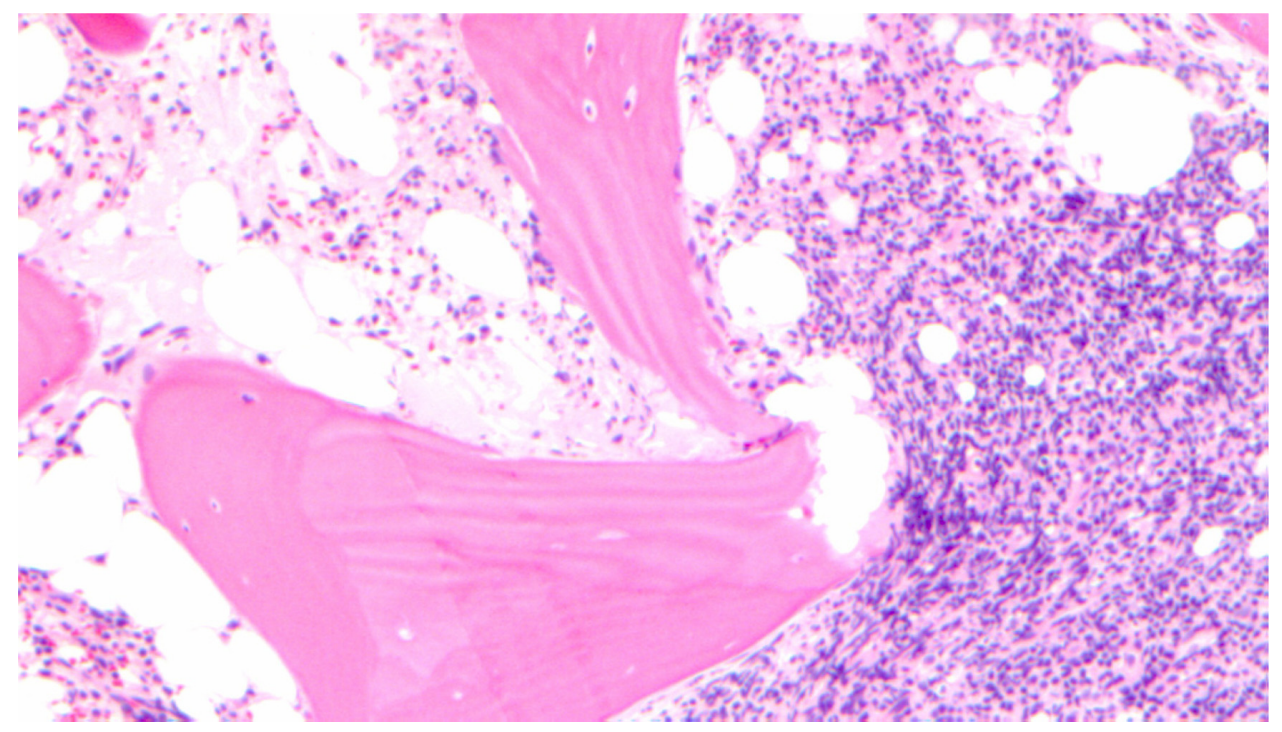

Figure $3 \mathrm{~A}$ small interstitial lymphoid aggregate is identified (magnification, 100x).

pathologic correlation. Patient was started on CLL-directed therapy with Rituximab $375 \mathrm{mg} / \mathrm{m}^{2}$ and Cyclophosphamide $1000 \mathrm{mg} / \mathrm{m}^{2}$. After only two cycles, the patient experienced a dramatic improvement in his symptoms. The muscle strength and swallowing improved significantly. Also, we noticed disappearance of his skin rash which had persisted despite previous treatment with topical and systemic steroids. The patient was able to swallow and subsequently his percutaneous endoscopic gastrostomy tube was removed. The patient completed six cycles of chemotherapy and at the end of therapy he was able to stand, squat, and walk independently with marked improvement in strength both proximally and distally.

\section{Discussion}

CLL is frequently complicated by the development of autoimmune complications which mostly involve the components of the hematopoietic system. Autoimmune hemolytic anemia is the most common of these and its frequency ranges from $10 \%-15 \%{ }^{1}{ }^{1}$ Autoimmune thrombocytopenia, pure red cell aplasia, and Evans syndrome ${ }^{2}$ are much less frequent. ${ }^{1,3}$ Nonhematological autoimmune phenomena are rare. There are various reported cases of rheumatoid arthritis, systemic lupus erythematosus, Sjögren's syndrome, Grave's disease, ulcerative colitis, pernicious anemia, nephritic syndrome, pemphigus in association with CLL.

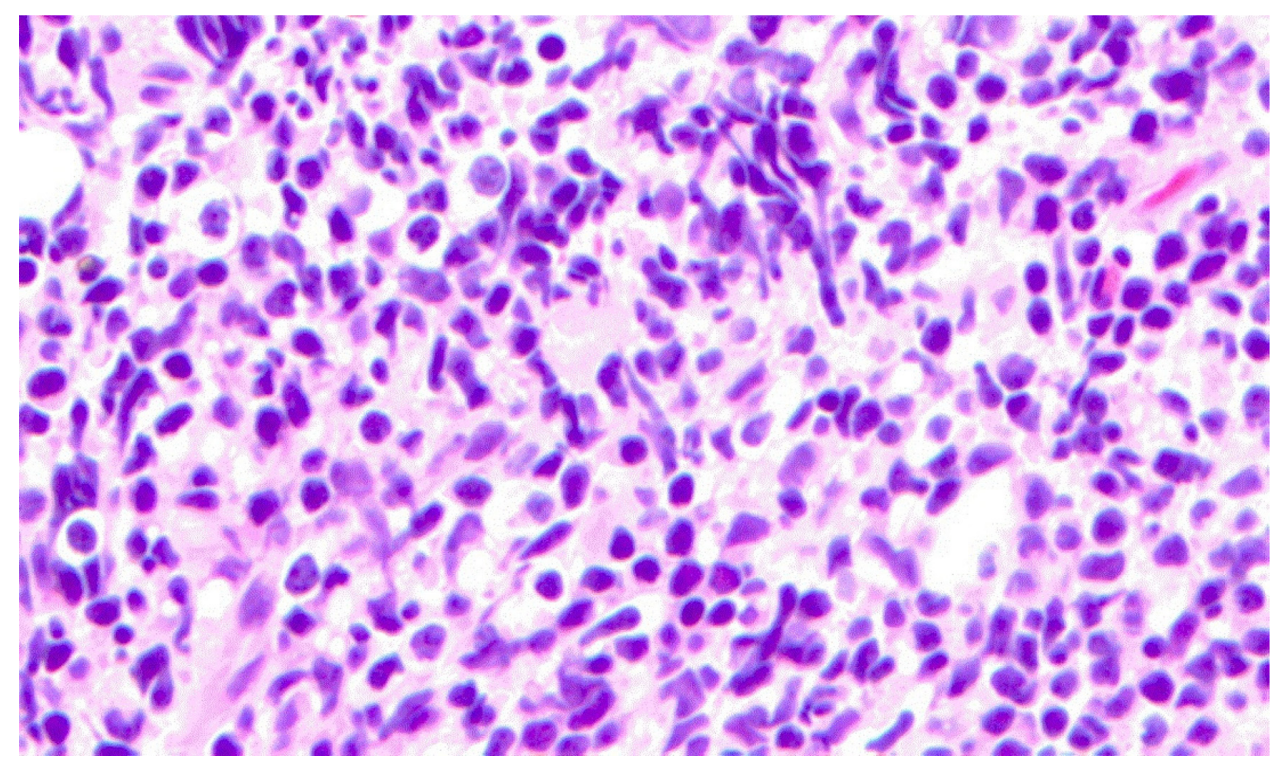

Figure 4 Monomorphous small lymphocytes with scant pale cytoplasm and condensed chromatin (magnification, 400x). 


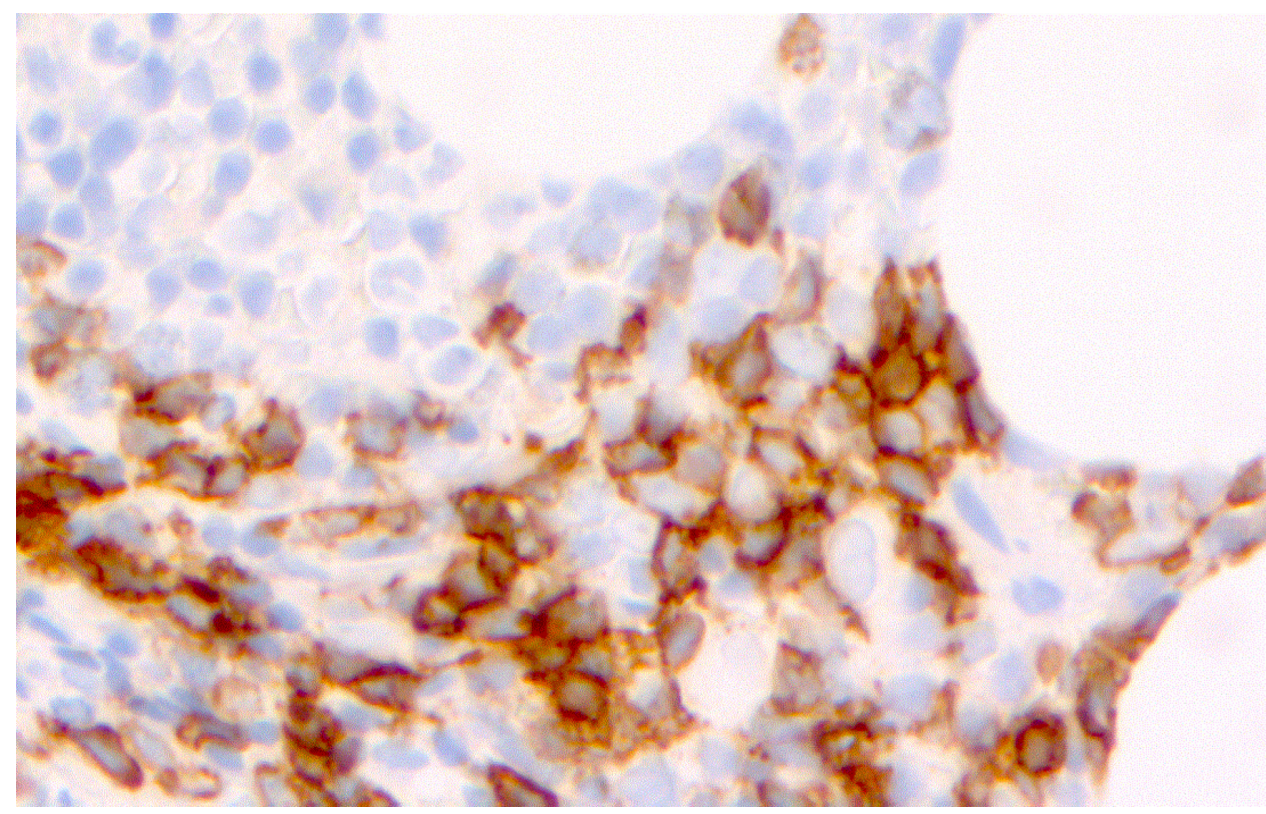

Figure 5 CD5 positivity (magnification, 400x).

Neuromuscular complications in association with CLL commonly presents with demyelinating neuropathy. To our knowledge, this is the first reported case of neuropathy combined with myopathy and dermatitis together in a patient with CLL. Also, timing of the onset of clinical symptoms is another atypical facet of our case. In all previously reported cases, neuromuscular complications developed later in the course of the disease after the diagnosis of CLL had already been established. In this case, the symptoms heralded the diagnosis without significant lymphocytosis for over a year, which posed a significant diagnostic challenge.

Neuromuscular disorders have been infrequently described in the setting of CLL. This is partly because of difficulty in establishing a clear association between the two. This was also the case in our patient as symptoms of demyelination and myopathy were without any significant lymphocytosis which had masked the diagnosis for over a year. Sometimes, the two diagnoses are considered to be coincidental. CNS infiltration by leukemic cells was first demonstrated

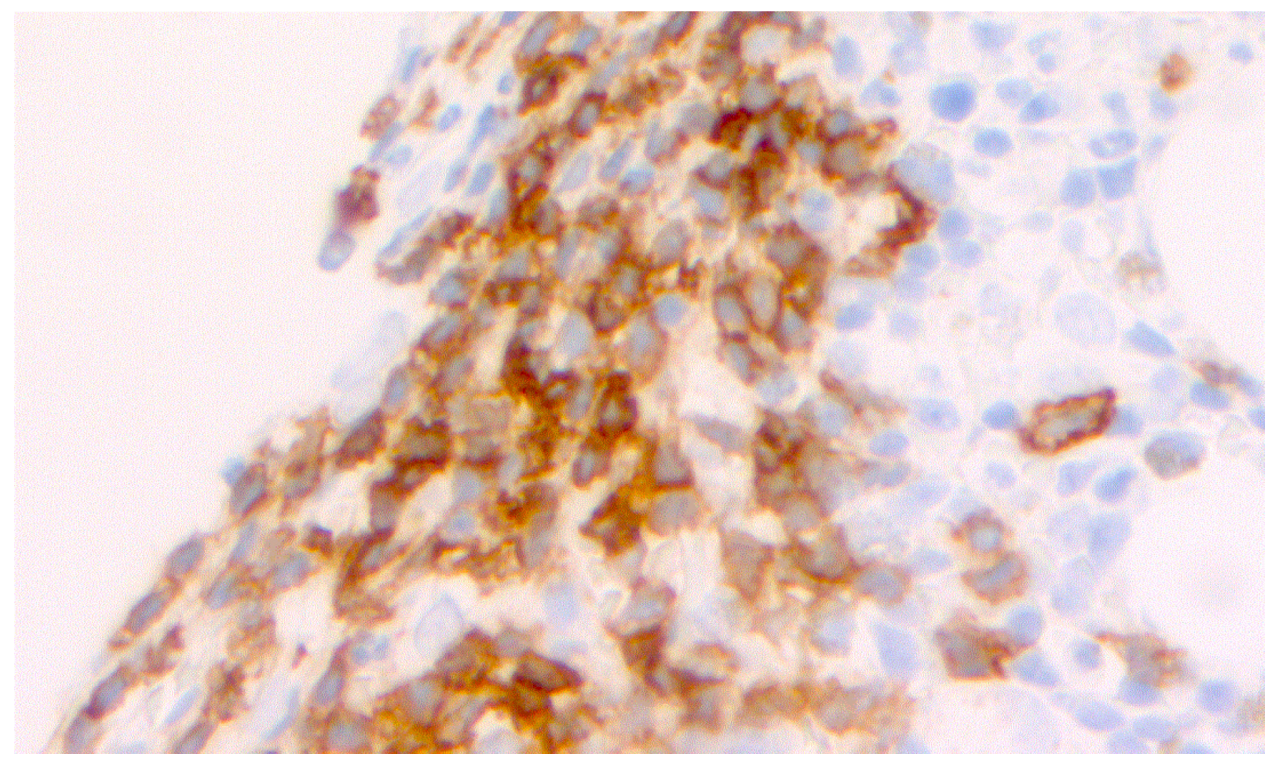

Figure 6 CD20 positivity (magnification, 400×). 


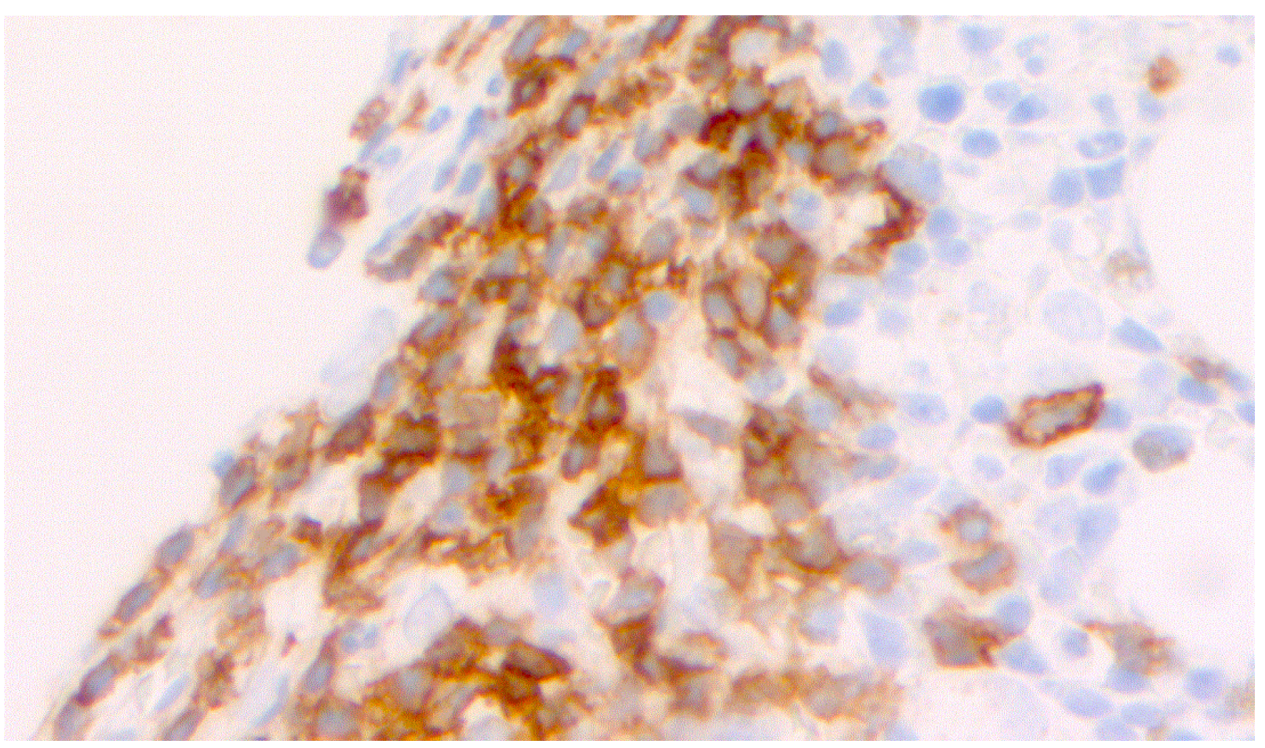

Figure 7 CD23 positivity (magnification, 400×).

in 1983 in an autopsy series. Eight out of 16 patients with CLL had evidence of CNS involvement. In 1996, Creange et al demonstrated the clonal origin of infiltrating cells by polymerase chain reaction (PCR) analysis. In this series, seven patients with CLL and diagnosis of neuromuscular disorder were evaluated by imunohistochemistry and PCR analysis of the biopsy specimens. All patients had evidence of monoclonal B-cells in the nerve or muscle tissue. ${ }^{4}$ However, the mere presence of monoclonal cells within the tissue still does not answer the primary question: Is the leukemic cells infiltration the primary event in the pathogenesis of neuromuscular symptoms or are the leukemic cells just recruited along with other inflammatory cells?

There are several hypotheses regarding the pathogenesis of autoimmune phenomena in CLL. Most of the research encompasses hematological complications. There is paucity of data regarding the pathophysiology of neuromuscular complications. Earlier research focused on autoantibody production. Sthoeger et $\mathrm{al}^{5}$ demonstrate the production of autoantibodies by neoplastic B-cells. Their work revealed the presence of antibodies in the sera of CLL patients with the same light chain sub-type as the surface immunoglobulin M (IgM) of neoplastic cells. Later work showed that the same light chain subtype is present in only $50 \%$ of CLL patients with autoimmune hemolytic anemia. ${ }^{6}$ However, most cases of CLL produce little immunoglobulin. Therefore, autoantibody production fails to explain the autoimmune complications in the majority of cases.

Another explanation is the dysregulated immune system in CLL patients. Development of autoimmune hemolytic anemia after treatment with agents like fludarabine is thought to be the result of the loss of T-cell regulatory control after chemotherapy. Purine analogs can cause long lasting depletion of CD4 cells and the development of autoimmune phenomenon may be delayed as long as 10 years. ${ }^{7}$ Also, the clonal B-cells in patients with autoimmune complications express an activated phenotype compared to the patients without autoimmune phenomena. There is higher expression of CD38 and FMC7 on the cell surfaces in CLL patients with autoimmune phenomenon. ${ }^{8}$

Intravenous immunoglobulin (IVIG) is used in the treatment of several autoimmune complications associated with malignancies including paraneoplastic syndromes. The response to IVIG is reported in certain cases of paraneoplastic syndromes, eg, paraneoplastic cerebellar degeneration; $;{ }^{910}$ pediatric opsoclonus; ${ }^{11}$ and some cases of stiff-person syndrome. ${ }^{12}$ In contrast, there is no data reporting the effectiveness of IVIG in CLL associated neuropathy and myositis. Lack of response to IVIG argues against autoantibody as the sole factor in disease pathogenesis. Alternatively, there is a possibility of permanent neurological damage in which case it should not respond to any therapy. Substantial improvement in neurologic symptoms with CLL directed therapy suggests against permanent neuronal damage.

\section{Conclusion}

This case highlights two important findings in the clinical course of CLL. First, the autoimmune complications can occur without significant lymphocytosis in the peripheral blood. Second, paraneoplastic autoimmune phenomena can be the initial presentation of hematologic malignancies. 
The clinicians should be aware of these, as early diagnosis can prevent significant morbidity associated with these disorders.

\section{Disclosure}

Written informed consent was obtained from the patient for publication of this case report and accompanying images. A copy of the written consent is available for review by the Editor-in-Chief of the International Journal of General Medicine. The authors declare that they have no competing interests in this work. SH and MP followed the patient during his several hospitalizations and in the outpatient clinic. MO analyzed and interpreted the patient data regarding the diagnosis, and followed the patient during his chemotherapy in the oncology clinic. EY interpreted the EMG and the nerve conduction studies and followed the patient from the neurology team in Staten Island hospital during his IVIG treatment. MW interpreted the histologic examination of the muscle biopsy from the pathology department in Staten Island Hospital findings. All authors read and approved the final manuscript. The authors wish to acknowledge Dr K Tanji of Columbia University Pathologists, Neuropathology Outreach Laboratory, New York, NY, for muscle biopsy study and expert interpretation.

\section{References}

1. Hamblin T. Autoimmune disease and its management in chronic lymphocytic leukemia. In: Cheson BD, editor. Chronic Lymphoid Leukemias, 2nd ed. New York, NY: Marcel Dekker; 2001:435-458.

2. Barcellini W, Capalbo S, Agostinelli RM, et al. Relationship between autoimmune phenomena and disease stage and therapy in B-cell chronic lymphocytic leukemia. Haematologica. 2006;91(12):1689-1692.
3. Zent CS, Ding W, Schwager SM, et al. The prognostic significance of cytopenia in chronic lymphocytic leukaemia/small lymphocytic lymphoma. Br J Haematol. 2008;141(5):615-621.

4. Creange A, Theodorou I, Sabourin JC, Vital C, Farcet JP, Gheradi RK. Inflammatory neuromuscular disorders associated with chronic lymphoid leukemia: evidence for clonal B cells within muscle and nerve. J Neurol Sci. 1996;137(1):35-41.

5. Sthoeger ZM, Wakai M, Tse DB, et al. Production of autoantibodies by CD5-expressing B lymphocytes from patients with chronic lymphocytic leukemia. J Exp Med. 1989;169(1):255-268.

6. Centola M, Lin K, Sutton C, et al. Production of anti-erythrocyte antibodies by leukemic and nonleukemic B cells in chronic lymphocytic leukemia patients. Leuk Lymphoma. 1996;20(5-6):465-469.

7. Hamblin TJ. Autoimmune complications of chronic lymphocytic leukemia. Semin Oncol. 2006;33(2):230-239.

8. Duek A, Shvidel L, Braester A, Berrebi A. Clinical and immunologic aspects of B chronic lymphocytic leukemia associated with autoimmune disorders. Isr Med Assoc J. 2006;8(12):828-831.

9. Widdess-Walsh P, Tavee JO, Schuele S, Stevens GH. Response to intravenous immunoglobulin in anti-Yo associated paraneoplastic cerebellar degeneration: case report and review of the literature. $J$ Neurooncol. 2003;63(2):187-190.

10. Phuphanich S, Brock C. Neurologic improvement after high-dose intravenous immunoglobulin therapy in patients with paraneoplastic cerebellar degeneration associated with anti-Purkinje cell antibody. $J$ Neurooncol. 2007;81(1):67-69.

11. Wong A. An update on opsoclonus. Curr Opin Neurol. 2007;20(1): 25-31.

12. Pittock SJ, Lucchinetti CF, Parisi JE, et al. Amphiphysin autoimmunity: paraneoplastic accompaniments. Ann Neurol. 2005;58(1):96-107.
International Journal of General Medicine

\section{Publish your work in this journal}

The International Journal of General Medicine is an international, peer-reviewed open-access journal that focuses on general and internal medicine, pathogenesis, epidemiology, diagnosis, monitoring and treatment protocols. The journal is characterized by the rapid reporting of reviews, original research and clinical studies across all disease areas.

\section{Dovepress}

A key focus is the elucidation of disease processes and management protocols resulting in improved outcomes for the patient. The manuscript management system is completely online and includes a very quick and fair peer-review system. Visit http://www.dovepress.com/ testimonials.php to read real quotes from published authors. 